\title{
Superficial cerebral hemosiderosis in a patient treated with warfarin
}

\section{BMTP Nawasiwatte ${ }^{1}$, A T Sarathchandra ${ }^{2}$, T Rajapakse ${ }^{2}$, PS Gunaratne ${ }^{1}$}

Ceylon Medical Journal 2014; 59: 149-150

A 59-years old man presented with worsening chronic ataxia and recent onset dysarthria, deafness and dementia of three months. Over five years, he was investigated for unsteady gait and found bilateral cerebellar and pyramidal tract signs which were attributed to cerebellitis and transverse myelitis respectively. He already had an indwelling urinary catheter for suspected prostatism.

Ten years ago warfarin was commenced on him for a cardiac apical aneurysm and left ventricular dysfunction but recurrent haematuria and haemoptysis lead to withdrawal of it two years later. No past history suggestive of sub-arachnoid haemorrhage (SAH), head trauma or neurosurgeries were elicited. He had bilateral VIIIth nerve palsy and cerebellar signs. Spastic legs had extensor plantars but proprioception and sensorium were intact. Examination otherwise was unremarkable. Basic haema-tology, coagulation and biochemistry were normal and CT brain showed bilateral cerebellar atrophy with normal ventricles. The CSF was normal except for numerous erythrocytes. Audiogram revealed bilateral sensory neural hearing loss (SNHL) and T2W MRI brain and spinal cord showed hyposignal intensity along leptomeninges (Figures1 and 2). Patient was managed symptomatically.

Superficial cerebral hemosiderosis $(\mathrm{SCH})$ is a rare disease caused by deposition of haemosiderin in superficial and sub-pial layers of CNS due to repeated

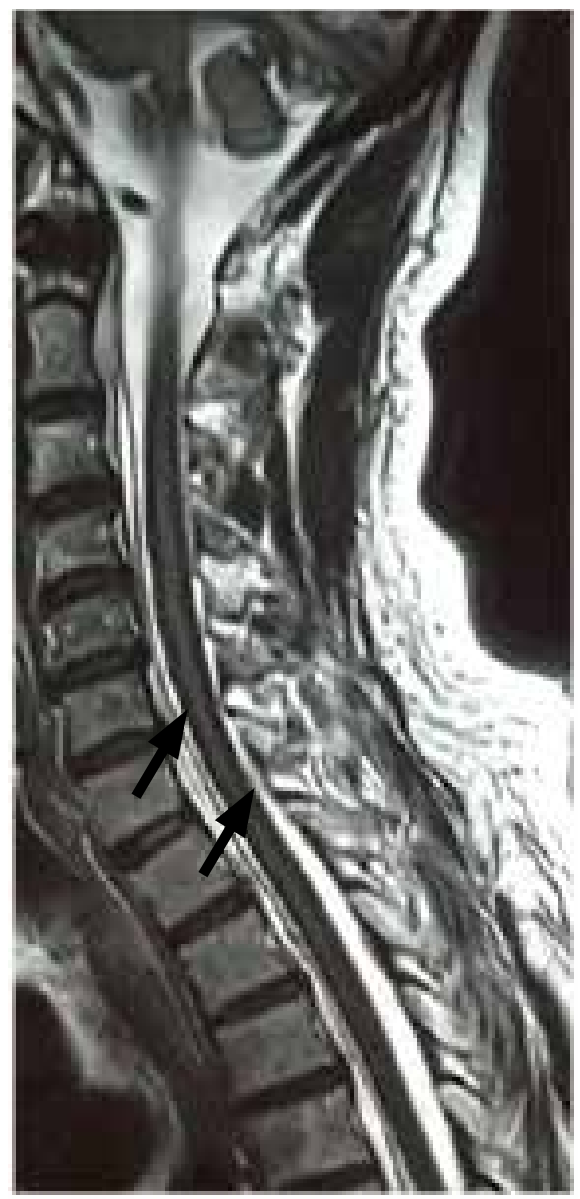

Figures 1 and 2. MR images of brain and spinal cord showing hypo-intensity of the marginal zones of CNS (black arrow head) indicating an iron induced susceptibility effect.

${ }^{1}$ Neurology Unit 2 and ${ }^{2}$ Department of Radiology, National Hospital, Sri Lanka.

Correspondence: BMTPN, e-mail: <thusha.nawasiwatte@gmail.com>. Received 13 May 2014 and revised version accepted 10 October 2014. Competing interests: none declared. 
chronic SAH or bleeding from CNS tumors and vascular malformations [1]. A single SAH is usually insufficient to result $\mathrm{SCH}$ and often a clinical SAH is unapparent and source of bleeding is obscure. Anticoagulants are a known but an uncommon association [2]. Classic symptom triad includes cerebellar dysfunction, SNHL and pyramidal tract signs but dementia and incontinence are common. Definitive diagnosis is by MRI, autopsy or neurosurgical findings of superficial hemosiderin deposition of CNS [3]. Usually the gap between onset of symptoms and diagnosis is many years. Hypo-intensity of CNS marginal zones on T2W MRI indicates an iron induced susceptibility effect which is pathognomonic for $\mathrm{SCH}$ [4]. Definitive treatment is by correcting the bleeding source. Iron chelators are ineffective.

\section{References}

1. Fearnley JM, Stevens JM, Rudge P. Superficial siderosis of the central nervous system. Brain 1995; 118: 1051-66.

2. Barreto RD, Ruano L, Cruz VT, et al. Superficial Siderosis and Anticoagulation Therapy: Different Presentations, Different Outcomes.Case Reports in Neurological Medicine 2012; http://dx.doi.org/10.1155/2012/745430 